\title{
Fostering a virtual community for lab animal scientists
}

\author{
Szczepan W. Baran, VMD, MS, President and Chief Operating Officer, \\ Veterinary Bioscience Institute, Piedmont Triad Research Park, \\ Winston Salem, NC; Course Director, Office of Professional Studies in the \\ Health Sciences, Drexel University College of Medicine, Philadelphia, PA.
}

Dr. Baran discusses his research interests and his work developing online meetings and courses for laboratory animal scientists.

\begin{abstract}
Your background in lab animal research includes the development of embryonic stem cell (ESC) lines for lab animal species. What are the research benefits of establishing these lines?

Unlike adult stem cells, ESCs can maintain their undifferentiated state despite repeated passages in culture and can differentiate into various cell types. They can be used to generate ESC-derived tissues for transplantation-based, regenerative therapies. In addition, ESCs can be used to generate transgenic animals. Derivation of ESC lines for canines, felines and nonhuman primates is important because these animals are vital biomedical models for heritable diseases and transplantation studies that have direct translation to human conditions. Heart cells or neurons derived from laboratory animal ESCs can be used for reliable screening of potential treatments to decrease the number of animals required for pre-clinical screenings.
\end{abstract}

You have also focused on the development and validation of rodent laparoscopic surgery procedures. How are these techniques used to improve the well-being of animals?

Rodent laparoscopy is an exciting area of research that fits into the $3 \mathrm{Rs}$ principles of refinement, reduction and replacement. It has been underutilized in rodents because of the lack of appropriate instrumention. However, with recent developments in this area, certain rodent laparoscopic procedures are now possible. Laparoscopy is less invasive and results in faster recovery for the animals. Serial biopsies can be collected from the same mouse or rat, which allows the animal to serve as its own control and reduces the number of animals used in a study. Furthermore, it can be used to inject cells and organisms into specific organs under magnification to develop neoplastic or infectious models with improved precision. Tissues and organs can be directly visualized. We are currently working on reproductive procedures that allow embryo transfer without externalization of the reproductive tract. These procedures are quickly gaining popularity and have been implemented within the laboratory animal industry.

What inspired your commitment to developing and providing online surgical training courses in laboratory animal medicine?

When I was a resident, my university was faced with the common challenge of how to provide surgical training to investigators, especially those working with rodent models. Online surgical training is available globally and on demand, has been validated in human surgery education and is a requirement during many surgical residencies. Online education allows people to further their education at an individual pace and breaks down international borders to provide access to experts across the globe. The laboratory animal medical community has greatly benefited from online training provided by AALAS Learning Library and Collaborative Institutional Training Initiative. Veterinary Bioscience Institute provides surgical training that is not available anywhere else.

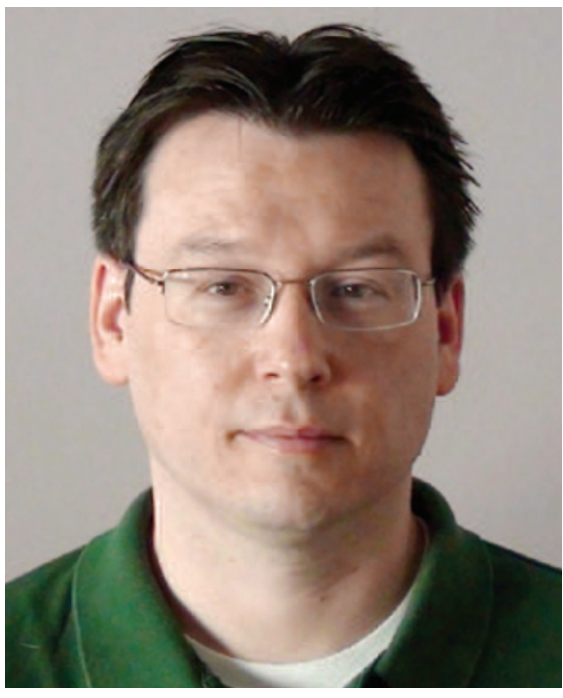

You also have been working with the company LabRoots to provide Online 2012 Laboratory Animal Science BioConference Live. What is the value of offering such a meeting online?

This exciting project is a dream realized, after four years of work and preparation. This conference will bring together global experts in laboratory animal science, with one major difference from other conferences: it is free to participants. This will allow us to provide education to all members of the laboratory animal community who want to learn, regardless of their allocated training budgets. Attendees will be given access to approximately 40 sessions at which they can question speakers and browse the virtual lobby, exhibit hall and exhibitor booths. Additionally, participants will be able to receive Continuing Education credits. Since this endeavor is specifically for the laboratory animal community, many affiliated organizations and vendors are supporting our effort. Organizations and individuals have joined us because the ability to share information on an international level, in a format that facilitates participation without the costs typically associated with scientific conferences, will be beneficial to the laboratory animal profession, biomedical researchers and laboratory animals themselves. 